\title{
Negotiating and Exploring Relationships in Métis Community-Based Research
}

\author{
Amanda LaVallee, Cheryl Troupe, Tara Turner
}

\begin{abstract}
Adding a Métis voice to the larger discourse on Indigenous (Métis, First Nation, and Inuit) health research, this work shares experiences and insights gained in relationship building from a community-based Métis research project entitled, Converging Methods and Tools: A Métis Group Model Building Project on Tuberculosis. A collaborative partnership between PhD student Amanda LaVallee, the Métis Nation Saskatchewan (MN-S) Health Department and two independent health researchers, the project, conducted from 2010 to 2012, incorporated a System Dynamics participatory methodology called Group Model Building (GMB), with Métis research methods, ethics, and knowledge, to build a model of tuberculosis (TB) experience in Saskatchewan Métis communities. This article examines the co-author's experiences with these collaborative methodologies and with the other partners in the research project, as well as the relational research stories that were essential to the practice of Metis community-based research. Moving beyond discussion of objectivity toward transparency about our presence within the research relationship, this work offers our collaborative experience as a success, and provides inspiration and insight on how to engage in ethical, competent, culturally appropriate, and relevant community-based research.
\end{abstract}

KEYWords Métis research, indigenous research, relational research, Métis communitybased research, and Saskatchewan Métis Research

In 2010, PhD student in Community Health and Epidemiology, University of Saskatchewan, Amanda LaVallee began meeting with Dr. Tara Turner, then Director of Health for the Métis Nation - Saskatchewan (MN-S), to discuss her proposed dissertation research. For the following two years, LaVallee, together with the MN-S Health Department and two independent health researchers worked toward incorporating a Euro-Western computer science-based participatory methodology called Systems Dynamics Group Model Building (GMB) into Métis research methods, ethics and knowledge, in the examination of tuberculosis (TB) in Saskatchewan Métis communities. ${ }^{1}$

\footnotetext{
1 System dynamics (originally developed in the 1950s) is a modeling paradigm for looking at systems and understanding dynamic problems. System dynamics takes a broad perspective of seeing overall structures, patterns and cycles in systems rather than seeing only specific events in the system. System dynamics models are built around a specific problem (for example: chronic and infectious diseases) (Sterman, 2000). Group Model Building is a participatory system dynamics method introduced in the 1980s. This method facilitates an inclusive, participatory, and collaborative effort of stakeholders in understanding and dealing with dynamic problems. Stakeholders are individuals, groups, or organizations who have an influence on or
} 
The MN-S is the governing body that represents Saskatchewan Métis people on political, social, and community issues. The health department in MN-S provides advocacy to help improve the health and wellbeing of Métis people in Saskatchewan. The department strives to improve the health status of Saskatchewan Métis people through a coordinated set of plans and actions that focus on community and stakeholder engagement, collaborative action, relationship building, data collection, research, and advocacy that are grounded in Métis understandings of community health and well-being (Métis Nation - Saskatchewan, 2012). Rounding out the research team was the then-assistant director of health for the MN-S, Cheryl Troupe, a Métis woman experienced in Métis community-based research, methods, ethics and protocols, as well as Karen Yee and Dr. Irini AbdelMallek, both experienced in Euro-Western research and methods, population health, system dynamics and GMB. ${ }^{2}$ Five years have passed since we embarked on this research. While we initially debriefed at the conclusion of our data collection, time has allowed us the opportunity to reflect on the process and outcomes of the research. As Métis community members and researchers, we have relied on reflexive practice and analysis through storytelling and story listening to inform this article. We have shared our experiences with community-based research and the challenges we encountered in merging Western and Indigenous research paradigms. We have integrated pieces of our stories from the original research (included in Amanda's dissertation) with our current reflections on the methods and ethics that guided us and on the relationships that were created.

As Métis community members and scholars, we understand that there are different kinds of knowledge and different ways of acquiring that knowledge. One can learn through theory and one can learn from the practical application of theory. Rather than have a theoretical discussion of our research process and methods for this paper, we chose to share the knowledge we gained through the practical application, and the specific techniques and tools that enabled us to have a successful research relationship and project. Some things can be learned only through experience; that is what we are sharing here.

\section{Culturally Responsive Research}

Métis peoples have existed at the margins of the Canadian historical, cultural, and social settings, and have been largely ignored as a distinct category in the production of most health statistics or in health research. This is due in part to failure of grant agencies to fund Métis-specific health research, as well as the lack of federal government responsibility for Métis health and well-being (National Collaborating Centre for Aboriginal Health, 2014). Even though Métis people comprise over thirty per cent of the total Aboriginal population in Canada, there is a clear and troubling under-representation of Métis-related research in the literature. Significant

will be influenced by a project or its outcomes. They are individuals who may be affected by decisions as well those that have the authority to make decisions such as managers, supervisors, front line workers, and community members (Vennix, 1996).

2 This project would not have been possible without the collaboration of Karen Yee and Dr. Irini AbdelMallek. While very important members of the original research team, they have not contributed to the co-authoring of this article due to their own personal and professional commitments. PhD research was completed and successfully defended in April 2014. To access completed dissertation refer to https://ecommons.usask.ca/bitstream/handle/10388/ETD-2014-04-1535/LAVALLEE-DISSERTATION.pdf?sequence $=5$

Engaged Scholar Journal: Community-Engaged Research, Teaching and Learning 
progress is required to learn about the health of Métis populations in Canada. Evans et al. (2012) outline four related, practical barriers to Métis community's health research: first, a lack of Métis-specific health care centers; second, limited human resources; third, reliance upon volunteers, which does not promote capacity building within Métis organizations; and fourth, political instability, which prevents long-term strategic planning and goal setting. Due to the challenges associated with obtaining appropriate and adequate health data indicators, we do not have a true picture of population health and well-being of the Métis in Saskatchewan. Accurate, adequate, and available research data on the health of the Métis population is needed to understand their health status and the disparities they face. Therefore, understanding health and wellness in Métis communities is critical in addressing health and health care disparities among Métis people; thus any research involving Métis peoples' health needs to be rooted in the community (Anderson \& Smylie, 2009).

As a team we desired to create capacity and knowledge about Métis health, research and methods; topics that have not been adequately addressed in academic literature (LaVallee, 2014). Therefore, in an attempt to add to the body of scholarly health research, LaVallee and the MN-S Health Department chose to study tuberculosis (TB) because Métis peoples have and continue to experience this infectious disease at disproportional rates compared to non-Indigenous peoples in Canada (Public Health Agency of Canada, 2006). ${ }^{3}$ Moreover, little is known, understood or published specifically about Métis people's past and present experiences of $\mathrm{TB}$.

Today, much research indicates that TB is heavily influenced by the social determinants of health, and is thus more prevalent in populations that experience racism, discrimination, poverty, lower education levels, overcrowding, poor water quality, and food insecurity (to name a few) (Public Health Agency of Canada, 2013). For these reasons, understanding TB in Métis communities requires culturally appropriate, responsive, and holistic research paradigms, methods, and ethics. Consequently, the GMB method was a means to begin community conversations and entry point in discussing the determinants of health impacting Métis peoples with regard to TB.

\section{Relationships, Respect, Relevance, Reciprocity and Responsibility}

Currently in Canada, most of the Indigenous health research methodologies and paradigm come from a First Nations and Inuit worldview, and Métis people are almost always considered part of that worldview. Past and current Indigenous scholars have been paving the way for an Indigenous research paradigm and methods to be recognized and utilized in our universities. Moreover, they are creating a body of Indigenous theoretical approaches, methods, protocols, and ethics in use by Indigenous researchers in the study of Indigenous peoples. The main

\footnotetext{
3 TB cases and rates in Canada indicate that TB among Indigenous peoples is higher than in non-Indigenous population within Saskatchewan (SK). The total SK Indigenous rate for reported new active and relapsed TB is 35.3 as compared with the non-Indigenous rate of 1.0 and a total Canadian-born rate of 8.1. Métis communities in SK reported an incidence rate of 19.9 per 100,000 compared to 7.3 per 100,000 across Canada (Statistics Canada, 2009).
} 
objective to date has been to ensure that research on and with Indigenous peoples is carried out in a culturally appropriate, respectful, ethical, truthful, responsive, and beneficial manner (Smith, 1999). Kirkness and Barnhardt (1991), Smith, (1999), Wilson (2008), and Kovach (2009) are a few of the influential and contemporary Indigenous/non-Indigenous scholars who have encouraged our awareness, knowledge, interest, and work in the field of Indigenous research. These scholars believe that Indigenous research is connected to the dismantling of the consequences of colonialism and is part of the self-determination process. Smith (1999), Wilson (2008), and Kovach (2009) agree that Indigenous sresearch should be rooted in Indigenous culture. Therefore, an Indigenous research paradigm reveals Indigenous values and beliefs, and therefore, Indigenous life (Smith, 1999; Wilson, 2008; Kovach, 2009). These scholars assert that "Indigenous methodologies tend to approach cultural protocols, values and behaviours as an integral part of methodology" (Smith, 1999, p. 15).

However, to date, there is little information on Métis-specific research paradigm and methods. Most of what we have learned in this project about a Métis research paradigm and methods has been through experience and by working with Elders and Knowledge Keepers in our community. As well, we (Amanda, Cheryl, and Tara) relied upon our individual and collective understandings as Métis women, and our experiences with conducting communitybased research; we also borrowed and adapted from numerous Indigenous research methods that were applicable to our project. We are keenly aware of the complexity and fluidity of Métis identity, ${ }^{4}$ which is not necessarily based on legal or bureaucratic terms. We understand that there is not one Métis identity, thus, not one Métis methodology, so we drew upon Indigenous methodologies in general. The methodologies we chose were grounded in teachings from our knowledge keepers, the relationship we formed with one another and the values of respect, reciprocity, relevance and responsibility.

Many Indigenous cultures in Canada and abroad believe that relationships are a vital part of our lives. Relationships not only involve people and places, but also the earth, sky, sun, moon, stones, plants, animals, spirits, ancestors, and the Creator. Central to this belief is awareness that all life is interconnected and that "we are all related." These relationships allow us to learn about ourselves, our families and communities, and the physical and spiritual world. Relationships are considered essential because they allow for the transfer of knowledge between individuals and generations (Kovach, 2009; NAHO, 2010; Settee, 2007; Wilson, 2008).

Creating and maintaining relationships is a process of personal growth and spiritual wellbeing. As Métis individuals, we have been taught that we must nurture the relationship that we have with ourselves in order to fully participate in relationships with others. This includes listening to our spirit and trusting our intuitions, values, beliefs, and morals. Being true to these teachings and ourselves allows us to have clarity, compassion, respect, and honesty with those around us. Engaging in relationships encourages listening, observing, and being present when participating with another person, people, or the environment. In a Métis research context, we

\footnotetext{
${ }^{4}$ See works by Chris Anderson, Michelle Reidger, and Brenda MacDougal who have all theorized and written on Métis historical and contemporary identities.
}

Engaged Scholar Journal: Community-Engaged Research, Teaching and Learning 
understand and appreciate that part of building relationships with individuals and community is dependent upon being present with all members involved. Creating space for relationships to develop includes introducing ourselves, and sharing with others our family and extended family histories - where we come from and what our connection to the community is. We have been taught that to build trust in a relationship we must share our physical, emotional, mental, and spiritual selves. Trust is maintained in a relationship by being true to our words, keeping our commitments, listening, and being consistent in our actions. Trust is established in what we say, how we verbalize our values, how we talk with others, and what we share about ourselves (LaVallee, 2014).

To respect means to "feel or show honour or esteem for someone or something; to consider the well-being of, or to treat someone or something with deference or courtesy" (Bopp \& Lucas, 1989, p. 76). Kovach (2009) argues that fundamental to any relationship in a personal or research context is the importance of respecting and valuing people and their knowledge. Respect develops in relationships grounded in connection, communication, transparency, honesty, and trust. Respect can be seen in specific actions and conduct, such as introducing people involved in the research, listening and observing, and allowing others to share about themselves, their families and experiences. Respect is also shown in the protocols through which we engage with others in research (NAHO, 2010). The offering of tobacco or a small gift to an individual demonstrates respect and is a non-verbal agreement that we will respect all individuals involved in the research by listening intently, being present, and honoring their presence as a community member, partner, collaborator and/or research participant. This protocol demonstrates that that we value their time, energy, and wisdom. In understanding Métis history and experience, we recognize that many Métis follow Catholic or Protestant religious beliefs and that the symbolic expression of respect, particularly the offering of tobacco, will look differently for these individuals. Elder Maria Campbell supports offering a small gift such as a bag and/or box of dried tea ${ }^{5}$ as an appropriate gift in showing appreciation and respect, as it is the gesture of offering that is important (M. Campbell, personal communication, January $10^{\text {th }}, 2012$ ). For our research we chose to offer tobacco in a small bag, decorated in a traditional Métis art form and made by a local Métis artist. This gift we felt was a demonstration of cultural pride, respect and a way of honouring our research, partners, collaborators and participants. With this gift, we also provided a small jar of homemade jam. We felt confident that this offering, together with the tobacco, would be respectful and appropriate (LaVallee, 2014).

Creating relationships and partnership with individuals and community members ensures they have an equal voice and participation in the research. This allows them to explore topics that are important, and therefore relevant to them (NAHO, 2010). Individuals and community members help to guide the research agenda, as well as ensure its accuracy through reading and writing aspects of the proposal, methods, and results before dissemination (LaVallee, 2014).

\footnotetext{
5 Offering a small gift is not limited to a bag or box of tea. It can be any gift, as it is the gesture that demonstrates respect, not the specific gift.
} 
Reciprocity is integral to Métis research. It is the building, nurturing and maintaining of relationships, not just between individuals and communities, or between the researcher and the community, but also with all of creation, including the land, sky, sun, moon, stones, plants, animals, spirit helpers, ancestors, and the Creator/God. It is based upon the understanding that we are connected to all things around us such that we should honour and give thanks to the air we breathe, the land we live on, and the resources that earth has provided for us to sustain our life. Reciprocity in a Métis research context may involve sharing stories, life experiences, events, and family history with individuals involved in the research - the act of storytelling and story listening. This is seen as an act of giving oneself physically, emotionally, mentally, and spiritually. However, as highlighted by Michell (1999), reciprocity can also be shown in symbolic forms such as giving tobacco to a research participant, collaborator, partner, mother earth, and/or the Creator. These reciprocal relationships can be seen as a sacred ceremony (Hart, 2010; Kovach, 2009; Wilson, 2008).

Responsibility and accountability are also fundamental to conducting Métis research. Engaging in research with a community means that we accept responsibility and accountability for the impact of the research on the lives of the community members with whom we will be working (Kovach, 2009; Wilson, 2008). Responsibility involves the assurance that we will work in an ethical way and be respectful of the community/organization and individual(s) that chooses to work with us. Wilson (2008) states, "The responsibility to ensure respectful and reciprocal relationships becomes the axiology of the person who is making these connections" (p. 79). Responsibility dictates that we must continually nurture the relationships we have created with individuals and with the community long after formal research has ended. We have a duty to uphold this kinship by maintaining contact with the community and helping if we are called upon (LaVallee, 2014).

\section{Relational Stories of Engagement: Reflection as Analysis}

As community members and research partners, we recognized the necessity of a strong research relationship as foundational to the success of our research. We chose reflexive practice to evaluate our work because in this instance we are analyzing our research relationship and not the actual outcomes of the research project. In doing so, we agreed that reflexive practice was well suited to the evaluation of our research relationship and that it aligned with our positionality as both community members and researchers. Reflexive practice is the dynamic process of critical reflection of the interaction within and between ourselves and our research partners, collaborators, participants, and the data (Guillemin \& Gillam, 2004). Reflexive practice appreciates that it is impossible for the researcher to remain outside of her own subjective being while engaging in research with individuals, collaborators and community (Creswell, 2003). As a result, we believe that using reflexive practice as an analytical technique allows us to contribute to community-based research methodologies for Métis research. To begin, we relate how we developed our research team, and then discuss relational stories with each other and the methods employed.

As a research team, we acknowledged and celebrated our diverse professional, educational, 
and cultural backgrounds. We recognized the need to establish personal and professional relationships with each other in order to build a cohesive and consistent research team. To do so, we engaged in weekly two-hour meetings over a seven-month period. During these meetings, we had to be precise in our intentions and goals, as well as create a relational space that honoured our social connections. It was our goal to build a relationship based on reciprocity, respect, relevance and responsibility. Meetings were held in a comfortable location, away from the institutional structures and offices of the research partners, allowing us to have dedicated and uninterrupted time for our relational processes. Amanda's house provided a friendly, open atmosphere that was removed from institutional parameters, designations, and authority. As a team we acknowledged that when we are at work or school, we might become employees and/or students first, and then individuals; and when we are in community, we are individuals first, and then employees and students. Sitting on a couch listening and watching a power point presentation on the television was very different than sitting in a boardroom in an agency or institution. Listening, laughing, learning, and sharing were transformed into a relevant relational context. How much, and what we shared, was very important in bridging our diverse cultural understandings as a collaborative and cohesive team (LaVallee, 2014).

For many of our meetings, team members took turns in the lead role of educator/ facilitator. Facilitation was the act of guiding the meeting process to respect people's time, create opportunities for equal participation, and to achieve the meeting goals. The first task on every agenda was sharing our perspectives, thoughts, and experiences personally and/or professionally. Each week Amanda emailed a meeting agenda to partners, which highlighted the meeting topics. Co-facilitation of the meetings created a power shift, allowing the student researcher to learn from others, and respecting the diverse areas of expertise and experience in the group. As well, sharing food at each meeting was a simple yet important gesture. In our cultural teachings, sharing food and drinks nurtures our emotional, physical, and spiritual beings. Food preparation and sharing is an expression of the symbolic importance of fostering good relations and creating a sense of community. The act of making and sharing food can be seen as a ceremony; it is a welcoming ceremony and bonding ceremony. Thus, each research partner and collaborator volunteered to bring food from their respective cultural backgrounds. Each consecutive week, the team member who brought food would also provide a story, teaching, and/or meaning concerning their food or culture (LaVallee, 2014).

Scheduling time, choosing a casual meeting location, sharing facilitation roles, as well as sharing our expertise, food and drinks, became the foundation for a cohesive, trusting, and transparent relationship among members of the research team. This relational work facilitated our co-creation of formal documentation necessary to meet the research and ethical obligations of the University of Saskatchewan, while also honoring the research ethics and protocols of the Métis community. Through these meetings, the research team was able to outline project research methods, ethics, knowledge translation, and dissemination. This documentation included a collaboration agreement, a memorandum of understanding (MOU) among research team members, and a research ethics application for the University of Saskatchewan Research Ethics Board. Over the course of seven months, the MN-S Research 
Team worked collaboratively to write all process documents. Although this took considerable time and effort, the process and the documents were built on the foundation of respect, reciprocity, and relevance.

\section{MN-S Health Department Relational Stories (Cheryl Troupe \& Tara Turner)}

To begin, we must emphasize that this research could not have been conducted had we not made the time and effort to build a strong relationship between Amanda and all members involved in the project. The MN-S Health Department understood that as health workers, educators, and researchers, we should not be working in isolation when it comes to Métis community health issues. Entering into this research, we recognized the value of strong research relationships and various experiences with different research projects and relationships.. At the outset we agreed that it would be fundamental to the success of the project to take whatever time necessary to build a relationship of trust, mutual respect, and responsibility. Therefore, each team member deliberately dedicated their time, attention, and self, entirely.

As a research team, we agreed that the proposed research methods were in alignment with a Métis research paradigm in that it privileged stories and valued holism and interconnections (all things are related). Initially it was our data collection plan to conduct a two-day group model building workshop where participants would share their TB stories and experiences that would then be translated into a causal loop diagram. ${ }^{6}$ The participants would work to sort the parts of their stories into positive and negative impacts and influences of TB. As a team, we spent much time learning about GMB methodology, sharing Indigenous methodologies and cultural understandings with non-Indigenous team members, and discussing the ways in which GMB aligned with and was challenged by Indigenous understandings and methodologies. As the team delved deeper into the group model building methodology and examined the tools and activities necessary to conduct a GMB workshop, we were confronted with a number of practical and intellectual challenges. We identified that a number of changes to the GMB method would be necessary in order to conduct the workshop and the research in a way that was meaningful, useful, ethical, responsible, and respectful for Métis communities.

Through our research relationship, we were able to trust and respect one another, to create a supportive environment where we were not afraid to question and challenge each other on issues as they arose. For instance, as the MN-S Health Department learned more about GMB, we became hesitant over the appropriateness of using such a method with Métis peoples. The workshop structure, with its prescribed roles and responsibilities seemed rigid and not conducive to the way in which stories are often shared in Métis communities. To us, it appeared that the process of documenting stories into a causal loop diagram could be disruptive and therefore disrespectful to the storyteller. As a result, the MN-S Health Department encouraged

\footnotetext{
${ }^{6}$ Causal loops are one way of diagrammatically representing a problem in the context of a system, and they involve capturing stakeholder stories, perceptions, experiences, knowledge, expectations, and conclusions about a systems problem (Albin, 1997). Causal loops consist of arrows connecting variables in a way that shows how one variable affects another over time. They are circular paths of cause and effect. Drawing a causal loop diagram is a good way to show how a change in one factor may impact another factor, which will then affect the first (Sterman, 2000).
}

Engaged Scholar Journal: Community-Engaged Research, Teaching and Learning 
Amanda to approach the workshop using a more Indigenous methodology, such as the Sharing Circle method. ${ }^{7}$ While we were confident that the Sharing Circle methodology would be more culturally responsive and appropriate, the MN-S Health Department initially received resistance from Amanda. Through the research relationship, we were able to challenge Amanda on her hesitancy in committing to Indigenous methods.

As the community partner, we noticed that Amanda often sought validation from her academic supervisor and co-supervisor, rather than trusting the expertise of community research partners. This resulted in the MN-S Health Department members feeling as though we did not always have an equitable partnership with Amanda. It seemed as though our opinions at times were not as valid as Amanda's academic supports. The privileging of Western academia over Indigenous ways, we believe had much to do with Amanda being a graduate student, trained in Western academic research methods, and the pressures to complete her doctoral degree. We understood that was important that she meet the requirements of her academic program, but we strongly encouraged her to trust the expertise, knowledge and wisdom of community, community practices, ethics and protocols. As community partners we wanted to nurture and mentor Amanda because we desired her to grow as a Métis woman grounded in her community, and in Indigenous research methods. While privileging Western methodologies and practices was certainly not Amanda's intention, we learned that Amanda was acting out of fear and her feelings of vulnerability; she knew the tension of being a Métis woman within a Euro-Western academic institution. Understanding her position, we were able to have very candid and often difficult discussions about the challenges and necessity in working to meet the rigor of the academy and the community. While confident that the research would be grounded in community ethics and protocols, we also needed to ensure that the research would be academically rigorous.

I (Tara) recognized the challenge of completing Métis research within a Western academic setting because of my own personal and academic experience completing my $\mathrm{PhD}$ a couple of years before. I understood this as a person who was not raised in a Métis community, and who had learned much about Métis identity through community connections gained in university, and also through my own $\mathrm{PhD}$ research on my Métis identity. Like Amanda, I was the first in my family to attend university. I saw myself in Amanda, recognizing both the opportunity, but also the challenges of being a Métis graduate student. I wanted to support her as a Métis person and researcher. When I was a graduate student, I felt extreme pressure both to conform to mainstream worldviews and to the other students, but also to act as a cultural representative for all Indigenous people. From my experience, Métis people, history, and culture were not well understood by many of my peers and my professors (if it is understood at all). This lack

\footnotetext{
7 Sharing Circles provide individuals the opportunity to share their personal stories, experiences, memories, thoughts, reactions, dreams, and feelings. In a Sharing Circle there is no beginning or end. Participants are neither first nor last. If they seat themselves in a circle, everyone can see each other. The circle establishes a safe non-hierarchical place in which all individuals have the opportunity to speak without interruptions. Without judgment or criticism, individuals share their stories and listen to others with their whole being: mind, body, heart, and spirit (Archibald, 2008; Hart, 2002; Kovach, 2009; Settee, 2007).
} 
of understanding has led to a lack of representation of Métis approaches to research within my field. As a graduate student, I was also struggling to gain firmer grounding in my own Métis identity and culture. It was hard to know how to move forward in a Métis way, how to explain to my committee so they would understand and support it, and how to stay true to my own growing identity as a Métis person. As academics and community members, we have all felt the pressure to privilege the academy over community. As Métis scholars and community members, we know that this academic environment can create extreme vulnerability in a new Indigenous researcher. I believe community and cultural supports can help the academic and personal journey be more fulfilling. Moreover, these supports have the potential to positively impact the cultural and personal identity, as well as create Indigenous researchers who will do engaged research with community.

I (Cheryl) came into this research project with more community research experience than either Tara or Amanda, so in many ways I was able to act as a cultural and community guide to the research team. Being a member of the Métis community in Saskatchewan and having worked with Métis communities for quite some time, I was confident in my experiences and knew that embarking on this project with Amanda would be an opportunity. In my own research experience, I have been fortunate to work with many knowledgeable cultural teachers and Old People that encouraged me to ground my work in community history, experience, culture and worldview. To me, embarking on this research with Amanda was an opportunity to share some of the lessons learned and challenges encountered in my own community-based research. I saw it as an opportunity to provide mentorship and support to Amanda, on her research journey. One of the most important lessons I wanted Amanda to realize was the importance of her Métis identity to the research. As Indigenous researchers, we have the responsibility, I strongly believe, to position ourselves within our research and address the multiple roles we fill as individuals, community members, and researchers. For that reason, I was willing to question Amanda on how this research process was intersecting with her own identity as a Métis woman, and as a member of community. And, because we had built a strong, trusting research relationship, I was not afraid to challenge her on the ways in which she was, (or was not) willing to incorporate her own understanding of Métis identity into the research. Often in the research process, I recognized Amanda's hesitance in fully embracing Indigenous methods (such as seeking guidance from an Elder and conducting a Sharing Circle). Through sharing our thoughts and feelings, I learned that Amanda was dealing with insecurity, vulnerability and the need to "measure up" as a member of the Métis community, and academia. In hindsight, I think that being honest, respectful and present in these discussions helped to alleviate some of these fears and insecurities, but also reaffirm and strengthen Amanda's identity as a member of our Métis community, and also bolster her confidence as a Métis woman conducting Indigenous research with and within community as well as within the Euro-Western academy.

At the time of this research, the MN-S Health Department had limited capacity in time and resources that we could commit to the research. However, we were willing to work with Amanda because we saw the value in supporting new Indigenous health researchers. We understood the long-term rewards of this, such as creating capacity in the field of Métis health

Engaged Scholar Journal: Community-Engaged Research, Teaching and Learning 
research, in the MN-S Health Department and within our own community. We chose to push forward with this research because we had the passion, drive, and grounding but also because we were in positions of authority within the MN-S to make this choice. We saw the value in the GMB method as innovative, with the potential to be flexible and inclusive in uniting Indigenous methods. As well, we saw the opportunity to challenge Euro-Western methods with Indigenous methods, and expand Euro-Western academic understanding of ways of conducting community-based research. Moreover, we wanted to be an example of how to more fully engage community, while demonstrating the value of Indigenous methods. We realized as the Health Department that we pushed Amanda harder than we might have pushed other researchers because she is a Métis woman, a Métis researcher, and we knew that her work would impact our Métis communities. We knew she was invested in us, her research, and community, and we felt the same level of investment in her. We knew that it was worth the risk to press her outside of her comfort zone, because our relationship with Amanda was strong enough to withstand challenges. We were driven by the desire to create an environment where researchers like Amanda can be members of their Indigenous community, and researchers grounded in their cultural identity.

Partnering with Amanda for this research project, the MN-S Health Department demonstrated that we were able to engage as full partners in Métis health research, and contribute to the knowledge available in Métis-specific population health research. The MN-S Health department was able to demonstrate the validity of a student researcher utilizing Indigenous methodologies within a mainstream Euro-Western institution. We could work with Amanda in a way that we feel was much more equitable than many of the research relationships we had previously been involved in. With limited capacity in terms of time and resources, we were able to use our relationships to create a team that provided the expertise and commitment required in completing this research. As diverse research team, we shared our academic, personal, and cultural worlds with each other, and we all learned and grew from our experience. By creating space and honoring Métis research methods, Amanda was supported in becoming a stronger Indigenous researcher.

\section{Student Researcher Relational Stories (Amanda LaVallee)}

Through extensive and sometimes hard conversations with Cheryl and Tara, I realized that my academic training and personal assumptions with regards to research legitimacy, validity, reliability, and rigidity in conducting "proper" scientific research was getting in the way of listening to my community partners and collaborators. I feared that if we engaged in Métis health research and incorporating Métis methods within group model building, the academy, the population health field, and the GMB community would see our methods as unscientific and invalid. During this time I was privileging my Euro-Western academic knowledge over my Métis knowledge. I did this because I was extremely insecure in my own being, feeling as though I was not Métis enough to engage in Métis methods. I felt as though my fair skin and education disenfranchised me from my Métis knowledge and culture. As a Métis scholar living and working in my community, I have been faced with tensions between our community 
knowledge and my academic training. I felt a consistent struggle between my feelings of legitimacy within my community and those within the academy. I was constantly negotiating Euro-Western and Métis knowledge. I was terrified about what my community might see or think of me; and I also felt the overwhelming pressure to complete and successfully pass my dissertation. I was fueled by the fear of what other people thought of me, the potential judgment of others, and the fear of the unfamiliar (Métis research methods: for example, Elder guidance and Sharing Circles). I felt completely vulnerable. For me, this was about the uncertainty, risk, and emotional exposure of being Métis in a Euro-Western system. I was taught that vulnerability means weakness. However, I realize now that vulnerability is about having the strength to be completely me. Being honest, transparent, revealing, trustworthy, accessible, and reliable are the foundations of building strong relationships (LaVallee, 2014).

Tara and Cheryl often had conversations with me about the extent to which I was willing, as a Métis woman and as a health researcher, to trust in the strength of my identity, my community, and to challenge myself to overcome my own hesitancy, fear and vulnerability. They encouraged me to not just observe the protocols I was comfortable with, but to seek out guidance from my cultural teachers, as well approach an Elder for guidance. What I discovered was that seeking support and guidance from a Métis Elder was integral to the research process.

I was anxious to approach and talk with Maria Campbell because I had never asked for guidance and support from an elder before. I knew that working with an elder meant a level of responsibility, accountability, and availability for and within my community that I was unsure I was ready for. I have known Maria Campbell for many years, and I have attended marriage and naming ceremonies where she was the ceremonial elder, conferences and workshops where she was the keynote speaker, and a graduate course where she was the professor. Although I had met her numerous times and have enjoyed our interactions and friendship, I was afraid that approaching her as an elder for guidance would open me to potential negative judgment. I was afraid that she would judge me as not being 'Métis enough' and question my community involvement. Moreover, she might disapprove of the research. However, my fears were completely unjustified; Maria was forthcoming, welcoming, and supportive. She reminded me that we are friends, and that I could seek her guidance, support, mentorship, and friendship at any point. Her knowledge and teachings were all grounded on the values of respect, reciprocity, relationships, and relevance. Seeking support and guidance from a Métis Elder was integral to my connections with community, my research collaborators, and as a Métis health researcher. In an effort to respect local Métis community protocols, culture, and values, Maria Campbell provided me with valuable lessons in honoring and appreciating diversity amongst our Métis communities, as well as how to create a culturally respectful and relevant GMB workshop (LaVallee, 2014).

As a student researcher, I felt immeasurable pressure from the institution to complete the research within the given time of four years. I was trying to progress quickly through the research; however, I continually underestimated the amount of time necessary to build trusting and transparent relationships with my community partners. I understand now that there should be no limits on the time it takes to building and maintaining relationships. However,

Engaged Scholar Journal: Community-Engaged Research, Teaching and Learning 
given the nature of academia and the work of completing a dissertation, time frames exist and these can dictate the research process, and, unfortunately, also the outcomes. For example, an ethics application can often be written in solitude by an independent student researcher, with support from her supervisor and/or co-supervisor within one day to a week. However, writing the ethics application with a community can take considerable time (within our project, seven months), given the nature of collaboration and giving choice and voice to the community. However, this is necessary if the partnership is to be equitable and mutually beneficial.

During the course of this project, we all had times of vulnerability in sharing our personal and professional lives. Being vulnerable was hard, but it was and is worth it because we shared laughter, tears, joy, happiness, anger, frustration and worry. Vulnerability and authenticity was at the root of my being Métis and doing Indigenous research and these were the glue that held our relationships together (LaVallee, 2014).

Overall, I learned some very valuable lessons; I learned that when I engage in relational research, I must be prepared to fundamentally alter any preconceived assumptions that I may have about my role in my community, in academia, and in research. I learned that relationships provide an opportunity for ethical enhancement by helping me to recognize my potential as a Métis community member and researcher. I believe all researchers need a community of people to share the joys and the struggles of research because solidarity can enhance research projects processes and outcomes (LaVallee, 2014).

This research is one example of Métis community-based research. Taking the time to create and strengthen relationships, as well as define the research process, methods, and ethics together, can result in the growth of knowledge and understanding for the researcher, for the community, and for the discipline and methods brought from Euro-Western academic traditions. Our research relational stories stand as a guide for both Indigenous and nonIndigenous researchers engaging in community research, and, more specifically provide encouragement to community organizations to partner with researchers to help build and shape the research they need for their communities and organizations, even with limited resources. When researchers and community members are engaged in relationship, there is the potential to create long-term, community-driven research agendas, that can sustain community research goals, as well as the goals and research agendas of the researcher.

\section{Conclusion}

Successfully conducting TB research with Métis people could not be accomplished through the standard toolbox of research techniques. We understand now that it was not one specific method or tool that characterized the success of our process: it was all of the Western and Métis methods and tools that collaboratively and synergistically worked together. If the outcome was the destination, our collaborative, relational process was the vehicle that got us there. Smith (1999) reminds us that "in many projects the process is far more important than the outcome. Processes are expected to be respectful, to enable people, to heal and to educate. They are expected to lead one small step further towards self-determination" (p.128). 
In taking the time to reflect on this research since its completion, we have gained insights into the process of undertaking community-based research from our personal experience, and have shared with academic researchers and Indigenous communities. We know that relationships built on trust, collaboration, and respect, are central to the success of this kind of research. Community-based research required commitment from everyone involved, and values partner with experience, capacity exchange, and expertise. Community-based research is successful when it is flexible in meeting the needs of the community, the researchers, and academia. And while community-based research is demanding, the benefits can far outweigh the challenges.

Our personal accountability was a humbling experience that helped us understand the colonial legacy of TB in our families and communities. We believe that health research requires collaboration among institutions, organizations, and stakeholders, dedicated to the health needs of Métis people. Engaging in community-based research that creatively merges research paradigms and methods, individuals, sectors, and institutions may help Métis communities to conceptualize and organize sustainable solutions to address health issues of importance (LaVallee, 2014).

In community-based research, researchers have the opportunity to build relationships with community members, not as research subjects or participants, but as individuals and communities. We have learned that doing community-based research has the potential to expand, stretch, and teach the researchers, collaborators, partners, and community members involved. This demanded vulnerability from each of us. However, it allowed us to learn about being researchers as well as being Métis community members within our research relationship. Overall, the relational foundation of this project was the key determinant of our success in conducting this Métis community-based research.

\section{About the Authors}

Amanda LaVallee (corresponding author) is a Red River Métis woman living in Coquitlam, British Columbia. She holds a Bachelor degree of Indian Social Work from the First Nations University of Canada, a Master's degree in Social Work from the University of Regina, and a $\mathrm{PhD}$ in the department of Community Health and Epidemiology (CH\&E) from the University of Saskatchewan. Currently, Amanda is a post-doctoral fellow in CH\&E, working on the research project Wuskiwiy-tan! (Let's Move!): Aging well in a northern Saskatchewan Métis Community. As well, contract faculty within the Child Family and Community Studies Department at Douglas College. Email: amaylavallee@hotmail.com 
Cheryl Troupe is Métis from Prince Albert, Saskatchewan. She holds a Master of Arts in Native Studies from the University of Saskatchewan and is currently a $\mathrm{PhD}$ candidate in history at the U of S, where she holds a SSHRC Doctoral Scholarship. Her dissertation entitled, “'They were hard working women, our Metis women years ago': Land Use in Qu'Appelle River Valley Metis Communities, 1880-1950," combines oral history methodologies with historical geographic information systems methods.

Tara Turner is Metis from a small community in the Kootenay region of British Columbia. Her PhD is in clinical psychology from the University of Saskatchewan. Tara's dissertation is titled Researching Metis Identity: My Metis Family Story. She is an assistant professor at the First Nations University of Canada, Saskatoon Campus, in the School of Indigenous Social Work.

\section{References}

Albin, S. (1997). Building a system dynamics model part 1: Conceptualization. Unpublished manuscript. Retrieved from http://clexchange.org/ftp/documents/Roadmaps/RM8/D-4597.pdf

Anderson, M., \& Smylie, J. (2009). Health systems performance measurement systems: How well do they perform in First Nation, Inuit, and Métis contexts? Pimatisiwin: A Journal of Aboriginal Health and Indigenous Community Health, 7(1), 99-115.

Bopp, J., \& Lucas, P. (1989). The sacred tree (2nd ed.). Lethbridge, AB: Four Worlds Development Press.

Creswell, J. (2003). Research design: Qualitative, quantitative, and mixed methods approaches (2nd ed.). Thousand Oaks, CA: Sage.

Evans et al. (2012). Funding and ethics in Métis community based research: The complications of a contemporary context. International Journal of Critical Indigenous Studies, 5(1), 54-66. Retrieved from http://ccgs.ok.ubc.ca/shared/assets/IJCIS44716.pdf

Guillemin, M., \& Gillam, L. (2004). Ethics, reflexivity and "ethically important moments" in research. Qualitative Inquiry, 10(2), 261-280.

Hart, M. (2010). Indigenous worldviews, knowledge, and research: The development of an Indigenous research paradigm. Journal of Indigenous Voices in Social Work, 1(1), 1-16.

Kirkness, V. \& Barnhardt, R. (1991). First Nations and higher education: The four R's - respect, relevance, reciprocity, responsibility. Journal of American Indian Education, 30(3), 1-15.

Kovach, M. (2009). Indigenous methodologies: Characteristics, conversations, and contexts. Toronto, ON: University of Toronto Press.

LaVallee, A. (2014). Converging methods and tools: A Métis group model building project on tuberculosis. (Doctoral Dissertation). Retrieved from: http://ecommons.usask.ca/bitstream/ handle/10388/ETD-2014-04-1535/LAVALLEE-DISSERTATION.pdf?sequence=5 
Métis Nation - Saskatchewan Health Department. (2012) Miyo âyâwin: Métis health and well-being strategy. Saskatoon, SK. Métis Nation -Saskatchewan.

Michell, H. (1999). Pakitinasowin: Tobacco offerings in exchange for stories and the ethic of reciprocity in First Nations research. Retrieved from http://www.sifc.edu/Indian\%20Studies/ IndigenousThought/fall99/tobacco.htm

National Collaborating Centre for Aboriginal Health (2014). Landscapes of First Nations, Inuit, and Métis health: An environmental scan of organizations, literature, and research, $3^{\text {rd }}$ Edition. Prince George, BC: National Collaborating Centre for Aboriginal Health.

National Aboriginal Health Organization (NAHO). (2010). Principles of etbical Métis research. Retrieved from http://www.naho.ca/documents/metiscentre/english/ PrinciplesofEthicalMetisResearch-descriptive_002.pdf

Public Health Agency of Canada. (2006). Tuberculosis in Canada - pre-release. Public Health Agency of Canada. Retrieved from http://www.respiratoryguidelines.ca/sites/all/files/Chapter\%201_ final.pdf

Public Health Agency of Canada. (2013). Canadian tuberculosis standards (7th ed). Ottawa, ON: Minister of Health. Retrieved from http://www.respiratoryguidelines.ca/tb-standards-2013

Settee, P. (2007). Pimatisiwin: Indigenous knowledge systems, our time has come. (Doctoral dissertation). Retrieved from http:/ /ecommons.usask.ca/handle/10388/etd-04302007-084445

Smith, L. (1999). Decolonizing methodologies: Research and indigenous peoples. Dunedin, NZ: University of Otago Press.

Statistics Canada. (2009). Aboriginal peoples survey, 2006: An overview of the health of the Metis population: Fact sheet. Retrieved from http://www.statcan.gc.ca/pub/89-637-x/2009006/art/art1-eng. htm

Sterman, J. (2000). Business dynamics: Systems thinking and modeling for a complex world. Boston, MA: Irwin McGraw-Hill.

Vennix, J. (1996). Group model building: Facilitating team learning using system dynamics. New York, NY: John Wiley \& Sons.

Wilson, S. (2008). Research is ceremony: Indigenous research methodologies. Black Point, NS: Fernwood Publishing. 